\title{
Applying Multi-agent Systems Coordination to the Diabetic Healthcare Collaboration
}

\author{
Peng Zhang, Guohua Bai, Bengt Carlsson, and Stefan J. Johansson
}

\begin{abstract}
Diabetic healthcare is characterized by the collaboration problem, which is manifested by problems of accessibility and interoperability. To improve the problem situation, we propose a Multi-agent Systems approach. The interactions among the diabetic healthcare actors are categorized on three levels: collaboration, coordination, and communication. Agents are designed to work on the coordination and communication levels, and support the collaboration among human actors. This paper presents a project Integrated Mobile Information Systems for diabetic healthcare (IMIS) to demonstrate how to apply Multi-agent Systems coordination to the collaboration among healthcare actors.
\end{abstract}

Index Terms - collaboration, coordination, diabetic healthcare, Multi-agent Systems

\section{INTRODUCTION}

$\mathrm{D}$ IABETES is a multi-disease that may lead to various complications. If not treated in time or in a good manner, diabetes may lead to serious consequences, including death. New technologies are appearing to help diabetic careproviders improving their work. Various kinds of medical devices are available in the market, e.g. digital glucose meters and digital scales. The medical data, i.e. glucose level, may be measured and sent to the server automatically from the devices via modems or gateways. There are already many such products in the market, e.g., Omron [13]. However, healthcare actors can only handle partial aspects of the diabetic healthcare, since the medical devices are still working in isolation. There is a need for an integrated system, which can provide a holistic view to the overall diabetic healthcare system and decision supports to the healthcare actors through synthesizing data from various sources, e.g., medical devices, nurses, personal assistants, doctors and patients.

Since diabetes can lead to multi complications and needs collaboration among healthcare actors, such a holistic view is important. It is required that healthcare actors from different medical fields collaborate together in the case of one diabetic patient. There is a need to develop a system that can collect and share data among those actors (both care-providers and care-receivers). In this paper, we present an e-Health project Integrated Mobile Information Systems for diabetic healthcare (IMIS), in which we introduce Multi-agent Systems (MAS) coordination to facilitate the collaboration among healthcare actors.

In the rest of this paper, we start by describing the collaboration problem in the diabetic healthcare system, and propose a MAS solution to it. Then we introduce our research project - IMIS to describe how agents are applied to the diabetic healthcare system. Finally, we give some discussions and a conclusion.

\section{The Diabetic HeAlthCARE System: THE PROBLEM OF COLLABORATION}

Healthcare system in general, and the Swedish diabetic healthcare system in particular, is characterized by being a multi-actor, widely distributed, and extremely complex system. From the perspective of patients, the system is too much segregated to get a holistic point of view. To well describe the problems, we would like to present a scenario first.

Linus, twelve years old, has diabetes of type one. One day he and his mother went abroad for tourism. The Mother carried with her some injectors for the daily insulin injections of Linus and some tablets as a backup. When she tried to give Linus the insulin injection on the last day before they went home, she broke the last injector by mistake. Thus, the Mother had no other choices but giving Linus tablets, which he had never taken before. She called the doctor whom Linus usually visited for suggestions. However, the doctor could not get the historical glucose record of Linus, since that record is on the database of Linus' school, which belongs to the County Council. So the doctor could not give a precise suggestion. For reasons of safety the doctor suggested taking one tablet. On his way home, Linus started to feel unwell. And when he managed home, he was sent to the hospital immediately.

The above scenario illustrates that the collaboration among healthcare actors, e.g., doctors, nurses, patients, relatives etc, is still problematic. To be more specified, the collaboration problem is manifested by problem of accessibility and interoperability of medical services.

\section{A. Accessibility Problem}

Two kinds of accessibilities in diabetic healthcare are identified, namely, physical service accessibility and information accessibility. Physical service accessibility means that medical resources should be available to healthcare actors anywhere at any time. To be accessible to the physical service no matter where and when, the relevant information must be 
accessible at that point of need, which is called information accessibility. In the above scenario, Linus and his mother were unable to reach the pharmacist. That is, the information accessibility was unavailable.

The details of the healthcare system vary from county to county. Hereby we take an example of the Blekinge province, which is located in the south of Sweden. The healthcare system in Blekinge is divided into three parts, namely hospital care, healthcare service and primary care. The responsibility is divided among two large healthcare actors, the County Council that is mainly responsible for medical and surgery related healthcare, and the municipality that is mainly responsible for elderly, disabled, and non-medical healthcare. As Fig. 1 shows, the hospital care is in the charge of the County Council. The non-medical related healthcare service is in the charge of the municipality. The primary care stands for the medical related healthcare that often needs long-term observation and cannot be taken at the hospital. For instance, the daily care of diabetes belongs to the primary care. After a doctor has made a diagnosis to a diabetic patient, the patient is transported to the municipality. For example, Linus, as a primary school student, stays at school most of the time. The school nurse, from the municipality, is in charge of his diabetic healthcare. Thus, the primary care is provided by both the County Council and the municipality.

The medical record of the patients, in most cases, cannot be shared between the County Council and the municipality, even though they intend to do so. The inaccessibility of information is called 'Collaboration Zones', which are indicated by the grey area between actors in Fig. 1. The patients live in the centre of the 'Collaboration Zones'.

\section{B. Interoperability Problem}

There are needs to harmonize the cross-border standards (both technical platform and information platform), terminologies, regulations, and business processes in diabetic healthcare. One patient often has to be transported among many divisions with many care-providers if he or she has more than one category of diseases, which is very often the case for diabetic patients. This demands an interoperable healthcare system where patients' relevant information can be accessed in a generally accepted standard. However, in the reality the healthcare providers have the records of patients in their own standards that are not operable by others. Interoperability is even more problematic when people are in another country or another county in Sweden. There are other issues that barrier interoperability, such as legalization of each organization, specified labor division, and even different culture and laws. In this paper, we touch only the issue of interoperability by using MAS technology to remove hindrance of sharing information.



Fig. 1. The information inaccessibility problem in 'Collaboration Zones'

\section{Multi-AGENT System AS A POSSIBLE SOLUTION}

To solve the above collaboration problem in diabetic healthcare, we need a solution that may help the healthcare actors and healthcare receivers accessing the correct information from various data sources (accessibility) in their understandable standards (interoperability) despite positions of users.

Agent technology, as our proposed solution, is now gradually reaching the front of application in healthcare area [12]. A software agent is considered as 'a computer system that is situated in some environment, and that is capable of autonomous action in this environment in order to meet its design objectives [17].' Some basic properties well discussed in the MAS community such as autonomy and coordination [15], are very amenable to the above mentioned problems. MAS has been applied to diabetic healthcare by various researchers in fields such as glucose monitoring and alarm [4, $9,10], \square$ biomedical control and management in diabetes [1, $2,8,11,14]$.

\section{A. A Holistic Diabetic Healthcare via MAS Coordination}

The accessibility and interoperability problems require a holistic view on the overall diabetic healthcare system. The information flows in diabetic healthcare are considered on three levels, namely collaboration, coordination, and communication. The three levels are organized in a way of hierarchy. That is, the collaboration consists of and is in charge of the coordination, which, in turn, consists of and is in charge of the communication.

\section{1) Collaboration}

The problems of accessibility and interoperability that are introduced above appear mostly on the collaboration level. The collaboration involves human factors. Motivations of various organizations are extremely complicated to be implemented in software agents. Although the computer agent 
is intended to perform intelligent actions, it is widely accepted that the agent intelligence is still far from the human intelligence. In general, the agent is considered intelligent if it is able to perform reactivity, pro-activity, social activity (simple interactions with other agents), and limited autonomy [16]. And this certainly cannot satisfy the needs of the reality. The collaboration among healthcare actors requires much more intelligent aspects than what agent technology can provide at this moment, e.g., some qualitative issues like motivations behind a series of activities.

In this research we define the collaboration as the interactions among diabetic healthcare actors in the real world. For instance, sharing the records of patients between hospital and healthcare services is considered as the collaboration between County Council and municipality.

We take as an assumption that the collaboration is done by people from healthcare organizations. Software agent only provides decision supports to the human actors, instead of decision making. When human decision makers decide what to do, they will transfer their preferences to agents. Each agent is assigned a task that should be performed either by itself or through coordination with other agents. When the task is finished, paused, or cancelled, agents should report to the decision makers or the upper level agents.

\section{2) Coordination}

The preferences transferred from the collaboration level include descriptions of the tasks/goals that agents should achieve. Often agents need to interact with others in order to accomplish a task. This interaction between agents is called MAS coordination. The roles and tasks of the agents are decided through coordination. After agents agree on their roles in performing the task, they take the responsibility to decide what measurable outcomes are expected, and how the outcomes are related to the tasks?

Coordination level is where agents exchange individual goals. The goals hereby mean the intentions owned by individual agents. Once an agent decides about its goal, it should commit to it. Thus, it is very important for the individual agent to coordinate their goals to avoid the conflicts to the lowest level. A possible solution is to carefully design in advance the sets of goals for agents to adopt. In the diabetic health care, this can be done through specifying agents' roles based on the healthcare labour divisions in needs specification.

\section{3) Communication}

The communication level is the technical level where agents exchange data and information in order to accomplish tasks. MAS coordination is realized through agents exchanging messages between each other. Agent messages contain valuable information, such as, agent goals, task information and so on. Agents in the same community should agree on the same ontology and codec so that they can communicate. Communication is considered as the fundamental skill that agents should possess. Some widely discussed Agent Communication Languages (ACL), for example, KIF [7], KQML [5], are suitable for the communication between agents.

\section{INTEGRATED MOBILE INFORMATION SYSTEMS FOR HEALTHCARE}

In out research project Integrated Mobile Information Systems for diabetic healthcare (IMIS), we developed an agent based systems - IMAS, for the diabetic healthcare for children. So far the first prototype of IMAS has been developed with JADE [3] framework to make it FIPA [6] compliant. The IMAS system works as an information coordinator that collects relevant information from various healthcare actors in different standards, manipulates them if necessary, and represents them back to the human actors in a common standard. Information flows in the IMAS contain the processes of collecting, manipulating and presenting relevant information. The relevant information may be medical data from different devices or agents, as well as the preferences that human actors transfer to the agents to accomplish tasks.

\section{A. IMAS System: from a holistic view}

Fig. 2 illustrates our holistic view on the diabetic healthcare system. In such a view, we distinguish the real world (human actors) and the MAS community (IMAS). The real world is considered to be on the collaboration level, where human actors collaborate in their work. The collaboration problem is described by conducting a field study with an outcome of user needs specification. The MAS community is on the coordination level. IMAS agents are designed to assist human actors in their work. The collaboration between human actors is enhanced through the coordination between agents. MAS coordination is regulated through predefined agent ontology and communication protocols, which are on the communication level.



Fig. 2 A holistic view on diabetic healthcare

\section{1) Collaboration}

Collaboration is supported through coordination and communication in the IMAS agent community - MAS. As Fig. 2 shows, the healthcare actors transfer their preferences to corresponding agents in the MAS. The MAS performs the 
tasks and reports the results to the actors for decision supports. The information flows of preference transferring and decision support concerns Human Agent Interaction (HAI), which is out of the topic of this paper.

Through user needs specification we defined the collaboration level. In IMAS, we defined three collaboration activities, glucose management, calendar arrangement, and task delegation.

- Glucose management. Diabetic patients usually care about their glucose level very much. They normally take measurement several times a day. With digital glumeters, the glucose levels can be read and transferred to a center database quickly. When the glucose level is too high or low, corresponding treatments must be taken by the patients or their care providers timely. Relevant information must be provided to the care providers in order for them to take the right actions. This process involves all the diabetic healthcare actors.

- Calendar arrangement. In acute situations, patients often need to visit the doctors or nurses. Usually the doctors and nurses are quite busy in their work. How to find an available time for the patients and their care providers to meet is another important collaboration activity. This also applies to the situation when the patients need to have a routine visit to the doctors. For example, small children with diabetes need to visit the doctors every three months.

- Task delegation. After doctors or nurses give diagnosis to patients, medical treatments are supposed to be taken. This can be done either by medical staff or the patients themselves, e.g., insulin injections. The medical treatments are decomposed to small tasks that are delegated to single human actors or software agents. When each task is done, results should be reported to the task manager. So far this part is partially finished. More efforts will be put on analyzing the work process of medical staff so that the task delegation will be clearly defined.

\section{2) Coordination}

In the first prototype of IMAS, we implemented four kinds of agents, PatientAgent, ParentAgent, HospitalNurseAgent, SchoolNurseAgent. As their names imply, they are working as personal assistants to the patients, parents, hospital nurses and school nurses correspondingly. IMAS agents have in their architecture Knowledge Bases, which stores Environment Model, local plans, and social context. The details of IMAS agent architecture will be described in another paper.

Herby we take an example of the glucose management to show how MAS coordination may help to enhance the diabetic collaboration via patient activity control, glucose monitoring, alarm and decision support.

We provide a new scenario about the same boy Linus. Linus now has in his body a sensor that measures his glucose level regularly and sends data to a database automatically. Linus has a mobile with IMAS Patient Control Panel installed on it. So each time Linus eats something or does some exercises, he will use his mobile to make a record, which will also be stored on the database. At the same time, on his mobile the PatientAgent works all time to monitor the glucose level of Linus, through reading the glucose records from the database. The glucose data is processed together with Linus' activities, which are recorded by the Patient Control Panel.

One day the PatientAgent detects that the glucose level is rather high $(12 \mathrm{mmol} / \mathrm{l})$. The PatientAgent reads from the activity records that Linus has taken some food that contains too much sugar and that he did not have any insulin injected lately. Based on the Environment Model stored in the Knowledge Base, the PatientAgent chooses two persons, Anna, the mother, and Christian, the school nurse, and sends them alarm messages with suggestion to give Linus an insulin injection, since they are probably close to him in this time. In this case, even though there are some other care-providers to Linus, for example, hospital nurses, they will not receive this alarm message. Nevertheless, a system log message will be generated and stored. So later all care-providers can see what has happened to the patient, if they want.

When the alarm message is received, the ParentAgent of Anna will automatically search for useful information about Linus and transform that to charts or other formats to present to Anna. She will confirm that the suggestion from the agents is convincible. Nevertheless, the decision is made by the human actors; the agents only provide relevant information to support the human actors in making good decisions.

3) Communication

The communication between IMAS agents is very simple. With JADE platform, we defined ontology and protocol for IMAS agents communicating with each other. The agent communication messages can be simple text messages in standard format, or be encrypted for reasons of security.

Besides, IMAS agents also communicate with human actors and relevant data sources, e.g., database server of patients' record. In the first case, graphical interfaces are provided to the users. Fig. 3 shows how patient may use the mobile to record his/her daily activities. Fig. 4 shows how the parent Anna receives an alarm from her working computer. 




Fig. 3 patient control panel (activity)

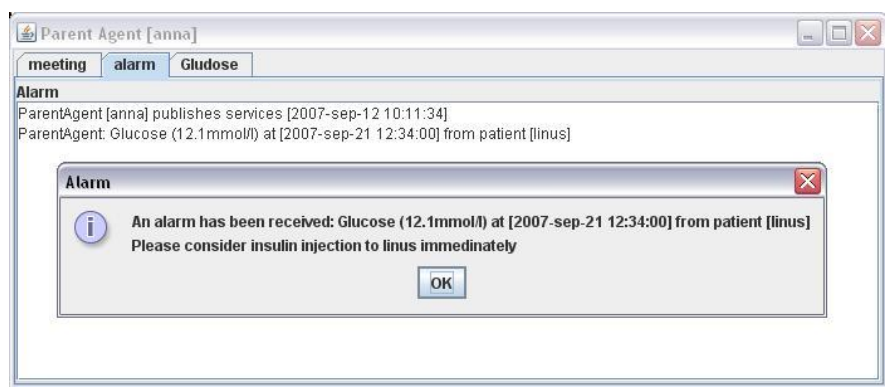

Fig. 4 ParentAgent receives an alarm

\section{DISCUSSION}

The IMAS system provides decision support to the human actors. We design and utilize computer agents as limited intelligent entities that provide their actors with important information, and are directly or indirectly engaged in collaboration activities.

\section{A. Facilitate collaboration with IMAS}

The accessibility of medical services can be very much improved with the IMAS agent coordination. IMAS agents may access to the medical data of patients at any time anywhere. Besides, the agents can process the medical data and present them as useful information for the actors to make decisions. For example, when detected an abnormal situation in the glucose level of diabetic patient, the agent will fetch relevant data and present to corresponding human actors with useful information for them to make a precise diagnosis timely.

Interoperability is another aspect that IMAS agents will solve. On the communication level, IMAS agents communicate with each other using predefined ontology. The IMAS ontology can be understood as a library of relevant concepts that are used in medical setting. The concepts are clearly defined and used in agent communication. The patient records are transferred between agents in the predefined standard - IMAS ontology, so that all the agents have will have the same understanding on one piece of data. Afterwards an agent will transfer the data to the corresponding standard that can be understood in the organization that this agent is working in. In this way, IMAS works as an interpreter that understands all the standards of different information resources.

\section{B. Security/Privacy}

Another important aspect that IMAS unavoidable should handle is the security and privacy. Although this is out of the main theme of this paper, we would like to give a brief discussion to it.

First, the operational security must be guaranteed. In a diabetic healthcare environment there are a lot of medical devices connected, relying either on human assistance and/or automatic registration. Alarm functions should be properly functioning and transmitted to responsible authorities.

Second, in a distributed information system it is vital for the system to guarantee an uninterrupted connection where it is possible to fully rely on transmitted data. In the healthcare environment the security of the information flow must prevent against interception, interruption, modification and fabrication. There need to be demands regarding confidentiality, integrity and availability regarding the information flow. Normally this means protecting data from unauthorized disclosure, alteration and destruction combined with verifying the source of data, e.g. using complex passwords, IP-Sec protocols and hash sums.

Third, sharing privacy sensitive medical data among various authorizations requires an extended security and privacy strategy. Normally the hospital or municipality is skilled at handling privacy issues within their domain, but what happens when new persons are involved and new agent-based techniques are introduced? Policies regarding security and privacy should be emphasized on and introduced equipments and agent-based functions should be examined.

Handling information sharing in a health care example normally involves both multi-level and multi-lateral security. In general, a doctor has access to all patient information where the primary care personnel only hold part of it. For a specific patient all relevant information should be distributed among all involved care-providers, i.e. invalidating part of the general restrictions. So, the MAS architecture should be designed with a proper privacy strategy that allows information sharing in the collaboration zone, but denies an outsider to do privacy collection.

\section{CONCLUSION}

We believe that an agent approach can improve the collaboration problem in the diabetic healthcare. A holistic analysis on the diabetic healthcare provides a three level view. On the collaboration level, the accessibility and interoperability problems are analyzed. The MAS coordination techniques are used to solve the accessibility problem. And the agent communication using predefined ontology can improve 
the interoperability problem.

\section{REFERENCES}

[1] Amigoni, F., et al., Anthropic Agency: a Multiagent System for Physiological Processes. Artificial Intelligence in Medicine, 2002. 27: p. 305-334.

[2] Bellazzi, R., et al., A Telemedicine Support for Diabetes Management: the T-IDDM Project. Computer Methods and Programs in Biomedicine, 2002. 69: p. 147-161.

[3] Bellifemine, F., G. Caire, and D. Greenwood, Developing Multi-agent Systems with JADE. 2007: Wiley.

[4] de Haan, G., O.B. Henkemans, and A. Aluwalia. Personal Assistants for Healthcare Treatment at Home. in the 2005 annual conference on European association of cognitive ergonomics. 2005. Ghania, Greece.

[5] Finin, T., Specification of the KQML agent communication language. 1993, DARPA knowledge sharing initiative external interfaces working group.

[6] FIPA, The Foundation for Intelligent Physical Agents. 2001, see http://www.fipa.org.

[7] Genesereth, M.R. and S.P. Ketchpel, Knowledge Interchange Format, Version 3.0 Reference Manual. 1992, Computer Science Department, Stanford University.

[8] Gómez, E.J., et al., Telemedicine as a Tool for Intensive Management of Diabetes: the DIABTel Experience. Computer Methods and Programs in Biomedicine, 2002. 69: p. 162-177.

[9] Hernando, M.E., et al. Multi-agent Architecture for the Provision of Intelligent Telemedicine Services in Diabetes Management. in the workshop on Intelligent and Adaptive Systems in Medicine. 2003. Prague, Czech.

[10] Hernando, M.E., et al., Intelligent Alarms Integrated in a Multi-agent Architecture for Diabetes Management. Transactions of the Institute of Measurement and Control, 2004. 26(3): p. 185-200.

[11] Li, M. and R.S.H. Istepanian. 3G Network Oriented Mobile Agents for intelligent Diabetes Management: a conceptual model. in 4th Annual IEEE Conf on Information Technology Applications in Biomedicine. 2003. UK.

[12] Nealon, J.L. and A. Moreno, Agent-Based Applications in Health Care, in Applications of Software Agent Technology in the Health Care Domain. 2003: Basel, Germany. p. 3-18.

[13] Omron, http://www.omron-healthcare.com/. 2007.

[14] Tian, J. and H. Tianfield, A Multi-agent Approach to the Design of an E-medicine Systems. LNAI, 2003. 2831: p. 85-94.

[15] Weiss, G., Multiagent Systems - a modern approach to distributed artificial intelligence, ed. G. Weiss. 1999: the MIT press, Cambridge, Massachusetts, London, England.

[16] Wooldridge, M., An Introduction to Multi-agent Systems. 2001: JOHN Wiley \& Sons Ltd.

[17] Wooldridge, M. and N.R. Jennings, Intelligent agents: theory and practice. The knowledge Engineering Review, 1995. 10(2): p. 115-152. 